\title{
Multiverse Ethnography: A Qualitative Method for Gaming and Technology Use Research
}

\author{
Veli-Matti Karhulahti ${ }^{1}$, Valtteri Kauraoja ${ }^{2}$, Olli Ouninkorpi ${ }^{1}$, Soli Perttu ${ }^{1}$, Jussi Perälä ${ }^{1}$, \\ Vilma Toivanen $^{1}$, Miia Siutila ${ }^{12}$ \\ ${ }^{1}$ Department of Music, Art and Culture Studies, Faculty of Humanities and Social Sciences, \\ University of Jyväskylä, Finland \\ ${ }^{2}$ Department of Media Studies, Faculty of Humanities, University of Turku, Finland
}

\begin{abstract}
Author Note
This paper has been peer reviewed and published in Journal of Gaming \& Virtual Worlds:

Karhulahti, V., Kauraoja, V., Ounikorpi, O., Perttu, S., Perälä, J., Toivanen, V. \& Siutila, M. (2022) Multiverse ethnography: A qualitative method for gaming and technology use research. Journal of Gaming \& Virtual Worlds, 14(1), 85-110. DOI: 10.1386/jgvw_00053_1
\end{abstract}

We have no known conflict of interest to disclose.

Correspondence concerning this article should be addressed to Veli-Matti Karhulahti, Department of Music, Art and Culture Studies, Faculty of Humanities and Social Sciences, PO Box 35, FI-40014, University of Jyväskylä, Finland. Email: vmmkar@utu.fi

Appendix 1: Sub themes.

Appendix 2: Case study codes.

Data and Preregistration: $\underline{\mathrm{https}: / / \mathrm{osf} . \mathrm{io} / \mathrm{j} \operatorname{cn} 3 \mathrm{k} /}$

\begin{abstract}
This article introduces multiverse ethnography as a systematic team-based qualitative method for studying the mechanical, structural, and experiential properties of videogames and other technological artefacts. Instead of applying the ethnographic method to produce a single indepth account, multiverse ethnography includes multiple researchers carrying out coordinated synergetic ethnographic work on the same research object, thus producing a multiverse of interpretations and perspectives. To test the method, 41 scholars carried out a multiverse ethnography on two videogames, Cyberpunk 2077 and Among Us. Explorative thematic findings regarding both titles are reported and methodological implications of multiverse ethnography are discussed.
\end{abstract}

Keywords: anthropology, ethnography, methods, qualitative research, technology 


\section{Introduction}

Research on digital gaming is at least partly established through autographic methods. In the early 1980s, David Sudnow's book-length study (1983) on Breakout utilized his personal experiences of solving the videogame's mechanics, thus representing phenomenologically oriented anthropology that has also been called 'autophenomenography' (Gruppetta 2004). A decade later, Julian Dibbell's (1994) account of a 'rape in cyberspace' - a journalistic narrative with some ethnographic components - became a much-cited example of the potential that qualitative methods have for describing the complex events that take place in virtual worlds. And indeed, with the proliferation in the 2000 s of online roleplaying games in particular, numerous large-scale ethnographic studies (e.g. Boellstorff 2008; Chen 2012; Pearce 2009; Taylor 2006) established a firm qualitative ground for understanding videogames and virtual worlds.

A persistent and occasionally fair criticism of ethnographic research - often carried out by one researcher - has always been that a single scholar, from their own biased perspective (as all perspectives are), is relatively limited when it comes to building a comprehensive understanding of a phenomenon (e.g. Atkinson \& Hammersley 1983; see Noble \& Smith 2015). This limitation also applies to research on gaming and related technologies, which are typically designed with various forking paths or user functions that make it difficult or impossible for a single ethnographer to grasp the full spectra of engagement.

Without trying to replace traditional ethnographic approaches but rather building on them and the idea of qualitative tandem research specifically (e.g. Sundén \& Sveningsson 2012), the present study introduces multiverse ethnography as a systematic team-based qualitative method for studying the mechanical, structural, and experiential properties of videogames and other technological artefacts. The benefits of the method are comparable, for instance, to the Delphi technique (e.g. Linstone \& Murray 2002) and group concept mapping (e.g. Rosas 2017), which employ participatory qualitative processes; to our knowledge, however, none of these previous methods involve coordinated videogame or technology-specific fieldwork, like multiverse ethnography does.

The next section presents the methodological procedure. This is followed by case studies, in which we apply the method to Cyberpunk 2077 and Among Us. Finally, we debrief and reflect 
on the method in order to provide recommendations for all labs and teams who might wish to apply multiverse ethnography in the future.

\section{Multiverse ethnography}

Instead of applying the ethnographic method to produce a single in-depth account, multiverse ethnography includes multiple researchers carrying out coordinated and synergetic ethnographic work on the same research object, thus producing a multiverse of interpretations and perspectives. The method is very resource- and time-intensive, and thus it is not suitable for small teams or solo researchers; however, its plurality of perspectives has the potential to provide a more diverse view, with several limitations that we discuss later. The methodological procedure is presented below and elaborated in practice in the next section.

a. Based on the research question(s), choose an object of study and the specific ethnographic means by which the researchers will engage with the object.

b. The participating researchers, a minimum of two but usually more, carry out ethnographic work and document their observations and experiences.

c. After the fieldwork is completed, fieldwork journals are exchanged between researchers and coded with the chosen coding technique or manual.

d. The codes are collectively analysed by appropriate means, such as thematic clustering or phenomenologically.

Each step is briefly elaborated on below.

a. Because of the subjective element of ethnographic research, multiverse ethnography is well suited for mapping out, for instance, the phenomenological diversity of how people experience playing or using a specific technology in different ways. The method applies equally well to documenting, for instance, the design structures of videogames, in which case researchers can apply an existing manual of structures to be documented or explored (e.g. Consalvo \& Dutton 2006; Elverdam \& Aarseth 2007). Because the method is essentially exploratory, it mainly applies to open research questions (e.g. 'What interaction mechanics does the videogame provide for players and what functions do they serve?') and is not well suited for hypothesis testing. 
Multiverse ethnography is based on a distinct application of the ethnographic method (see Boellstorff et al. 2012), here understood both broadly and narrowly as systematic autographic engagement with subjectively observed and experienced events documented as field notes. We use the terms 'broadly' and 'narrowly', as this understanding is simultaneously unspecific (not representing a single, precise ethnographic method) as well as limited (being focused on the researcher's own interpretations and excluding, for example, interview-driven ethnography). There are no 'right and wrong' ethnographic sub-methods that may (not) apply here. However, subjective engagement and field journals seem to serve as an optimal choice for many imaginable multiverse ethnographic studies; it allows a diversity of controlled perspectives. That is, each scholar can be introduced to research goals, data, and their analysis on a balanced basis.

b. The minimum number of ethnographers is two, but for the method to be useful and produce a rich diversity of accounts, multiple ethnographers should participate. The upper limit is defined solely by the team's resources; however, for ethical and practical reasons, it will be valuable for future research to develop size justifications by testing, for example, saturation points with specific objects of study in specific research contexts. An efficient solution in many cases may be to recruit a 'principal ethnographer' to carry out extensive fieldwork and be supported by 'associate ethnographers', who can supplement the former's findings (principalassociate design).

For a participating researcher, the core mechanic in multiverse ethnography is thus subjective, reflective engagement with the studied artefact, for a chosen period, from the chosen (or open) perspective. Although the present case studies concern gaming and virtual worlds, there is no reason why this approach could not be applied to other types of technology use, such as social media applications (see Meier \& Reinecke 2020). Following the above, the length of the ethnographic work depends on both the object of study as well as the specific goals.

c. It is important for the ethnographers to document their fieldnotes in manners and styles that are understandable by other readers: after the fieldwork, the ethnographers' journals are exchanged between researchers to be coded. This enables second-level analysis on the collected data, adding analytic validity without the problems that having a completely external coder (having no experience of the videogame or technology at all) would entail. In addition to the above exchange coding - and especially if the principal-associate design is applied - it is 
recommended for one team member to run a second coding round with all the data to assess overall interrater reliability and be informed about negotiable discrepancies.

The coding process can be fully open or guided with a manual (see Saldaña 2012). The latter is fitting for structural analysis, for example, if the team has decided which components they are aiming to chart. Researchers can also make use of coding processes inclined towards grounded theory (Strauss \& Corbin 1994), thematic analysis (Braun \& Clarke 2006), and other qualitative traditions (see Campbell et al. 2013). It would not make sense to claim any coding approach to be superior here, but future multiverse ethnographic studies may yield evidence on the fit of certain coding processes over others. As an added benefit, the process enables meeting current open science gold standards (Meyer 2018): the ethnographers write their fieldwork journals and carry out coding so that the data and materials can be shared publicly with other researchers for reuse and replication, with ethical and privacy concerns being considered (e.g. removing possible personal identifiers).

d. As the final step, the team will carry out collective analysis of the coded data. Because all researchers have participated personally in the ethnographic work and coding, the collective analysis will merge multiple expert perspectives on a conceptual level that is both abstract and detailed: everyone should be able to reflect on and relate to both the higher-level code families and themes that emerge (if such are mapped), but also a large part of the individual codes that represent the deeper domains of observation. Depending on the chosen research questions, object of study, and specific ethnographic/coding techniques, the team may choose fitting cooperative means for conducting this phase - ultimately completing a triple-interpretive process where the ethnographers interpret the object of study in a double hermeneutic cycle (Karhulahti 2012) and the team members try to make sense of the written interpretations of their co-ethnographers (see Arjoranta \& Karhulahti 2014).

It is possible to also analyse the journals without systematic coding; for instance, in case of indepth phenomenological analysis, it may be more productive to apply an iterative close reading of individual journals (cf. interpretive phenomenological analysis) (Smith 1996) and triangulate them with a semi-structured dialogue with the ethnographer (who is part of the team). In such scenarios, teamwork must be coordinated carefully with solutions that go beyond the present article. Communicating about phenomenologically oriented sense-making in a large 
team of researchers poses different challenges than coding, and we leave those challenges to be tackled elsewhere (see Smith et al. 2009).

In the next section, we apply the above methodological frame in practice, as we present a multiverse ethnographic study of Cyberpunk 2077 and Among Us.

\section{Case studies: Cyberpunk 2077 and Among Us}

The goal of the case studies was to pilot the multiverse ethnographic method and yield preliminary answers to various technical questions, many of which were discussed above. For instance, many pragmatic issues related to field note techniques, coding, and final analyses expectedly emerge when the research team consists of a dozen - or, as in our case, $41-$ researchers. To improve the transparency, validity, and communication of the research design within this prospective team, all procedures were preregistered in the Open Science Framework before the start of our study (https://osf.io/9zgb5). The study did not require a local ethics committee review.

\subsection{Research questions and participants}

The study was carried out as part of a three-month long university course 'Methods in Games Research'. A total of 42 students (BA and MA levels) enrolled for the course and 38 passed; a list of participants can be found in the acknowledgements. All students who passed gave permission to use their work in this study and share it for educational and research purposes. The students were also given the option to contribute as authors, and four volunteered.

All 38 students were instructed to choose either Cyberpunk 2077 or Among Us as a research object and engage with it ethnographically for a minimum of 30 hours. This could be done either by personally playing or by watching a selected live-streamer playing the videogame. A list of previous qualitative and theoretical gaming research was provided as compulsory readings at the start of the ethnographic work (Aarseth \& Grabarczyk 2018; Consalvo 2020; Iversen 2012; Malliet 2007; Miller 2008; Quandt et al. 2009; Snodgrass et al. 2018), and two lectures were given on the topic; these may have affected the observations. The guidelines for observations were left open. Everyone was free to document anything that they personally considered relevant, with the single inclusion criterion being that the documented observations 
had to be about the videogames that were studied. For instance, those who engaged the videogames via live-streams were explicitly told to not make observations about the streamer or the chat, but only about the videogame that was being played (not everyone followed this criterion). Everyone was instructed to take fieldnotes in their own style.

Due to the exploratory nature of this work, we had no explicit research questions beyond methodology testing. Specific guidelines about what to document or observe about the videogames were explicitly omitted; the idea was simply to map out what BA and MA university students in Finland consider worth documenting about Cyberpunk 2077 and Among $U_{s}$ in a 30-hour process of engagement. It is worth highlighting that the limited number of hours likely produced a bias for the earlier content of each videogame, which nonetheless is the very content that players of these titles interact with most. The final research materials were: 30 ethnographic journals about Cyberpunk 2077 and eight ethnographic journals about Among Us. The length of the journals ranged between 2-58 pages for each, and they were written in either Finnish or English. The data and materials are openly available for educational and research reuse (https://osf.io/jen3k/).

\subsection{Analysis}

We then applied the above-mentioned exchange coding: each student swapped their journal with someone else and carried out an open coding round for the swapped journal. The students were introduced to the coding procedure via guidelines in a lecture and written instructions with examples (based on Saldaña 2012). Because we did not have any preconceived research questions, no manuals or design structural models were applied. Rather, everyone coded the journal data openly and formed code families and thematic categories based on their centrality and hierarchical relations (see Categorization Example at https://osf.io/jcn3k/). To increase the validity of the coding process (the students came from many different fields with varying levels of academic experience), one researcher in the team read all the journals and assessed the produced codes and their families/themes, marking inconsistencies. The rest of the team transcribed the codes and their families/themes separately into an online platform for analysis. After removing flawed data and merging overlap, 1764 unique codes remained for Cyberpunk 2077 and 407 unique codes for Among Us. At this point, the former had 263 themes and the latter 63 themes. 
In the next phase, all team members met via three successive online meetings to discuss thematic synthesizing. By negotiating each theme one by one (see Syed \& Nelson 2015), we merged overlapping themes and removed those that did not occur more than once or twice. Some of the remaining themes were labelled 'sub-themes' of larger thematic areas, and we thus organized them accordingly and respectively for each videogame (see Synthesis Example at https://osf.io/jen3k/). The overall thematic structures of both videogames are illustrated as tables below, and a more detailed list of their individual codes is attached (Appendix 1).

\section{Cyberpunk 2077}

\begin{tabular}{|c|c|c|c|c|c|}
\hline Theme & Subjejective & Environment & Storyworld & Mechanics & Aesthetics \\
\hline Sub-theme 1 & Eqmotitong $\underline{s}$ & Locations & Interactions & Interactions & Tech motif \\
\hline Sub-theme 2 & Crititiciss $\underline{m}$ & Characters & Characters & Bugs & Bugs \\
\hline Sub-theme 3 & Intertextextualitity & Tech motif & Narratives & Play styles & Audio-visual \\
\hline
\end{tabular}

\begin{tabular}{|c|c|c|c|c|c|}
\hline \multicolumn{6}{|l|}{ Among $U s$} \\
\hline Theme & 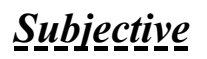 & Actions & Multiplay & Strategies & Design \\
\hline Sub-theme 1 & $\underline{N} / \underline{A}$ & Practices & Meta & Meta & Aesthetics \\
\hline Sub-theme 2 & $\underline{N} / \underline{A}$ & Mechanics & Communication & Personal & Changes \\
\hline
\end{tabular}

Table 1. Themes and sub-themes based on the openly coded content in 30 (Cyberpunk 2077) and eight (Among Us) ethnographic journals.

In Cyberpunk 2077, one of the large thematic categories did not concern the videogame as such, but rather the ethnographers' own subjective opinions regarding it - illustrated by documented emotions, criticisms, and intertextual comments (the latter often noting the videogame to be reminiscent of something rather than explicitly citing another cultural product). Because our case studies have a focus on videogames as such, we excluded the subjective thematic family from further analysis. The remaining four larger thematic categories - Environment, Storyworld, Mechanics, Aesthetics - concerned Cyberpunk 2077 as a 
videogame in particular. Four sub-themes were difficult to classify under a single category; hence, we positioned them with a dual identity as belonging to two separate larger categories (Figure 1).

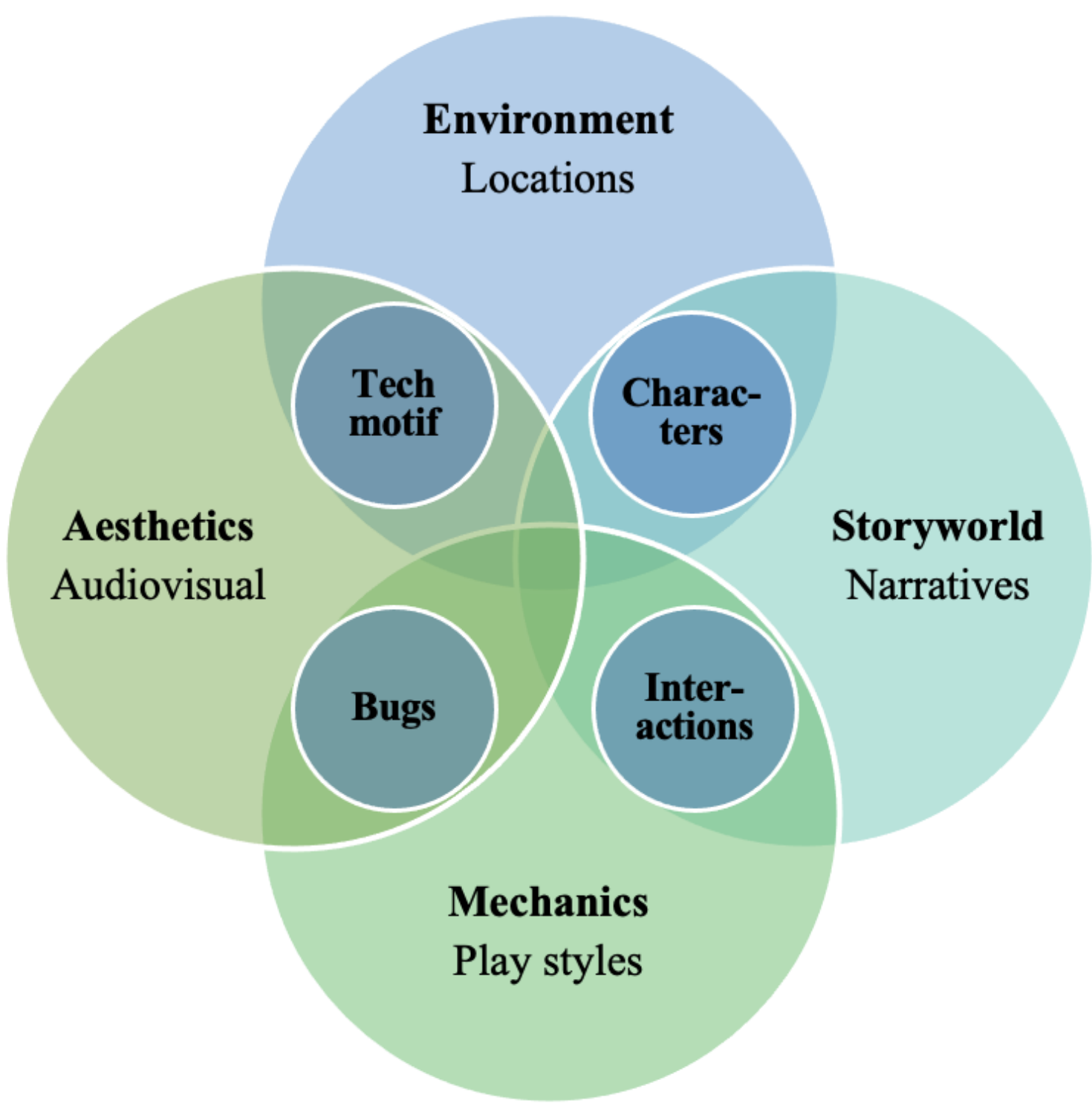

Figure 1. The exploratively formed structure of Cyberpunk 2077, based on the case study.

In Among Us, following the above, one large thematic family emerged around subjective observations about the videogame, and it was likewise removed. Four larger thematic categories remained: Actions, Multiplay, Strategies, and Design. We found two sub-themes for each thematic category, one of which we identified as belonging to two categories. The overall thematic structure is presented below (Figure 2). 


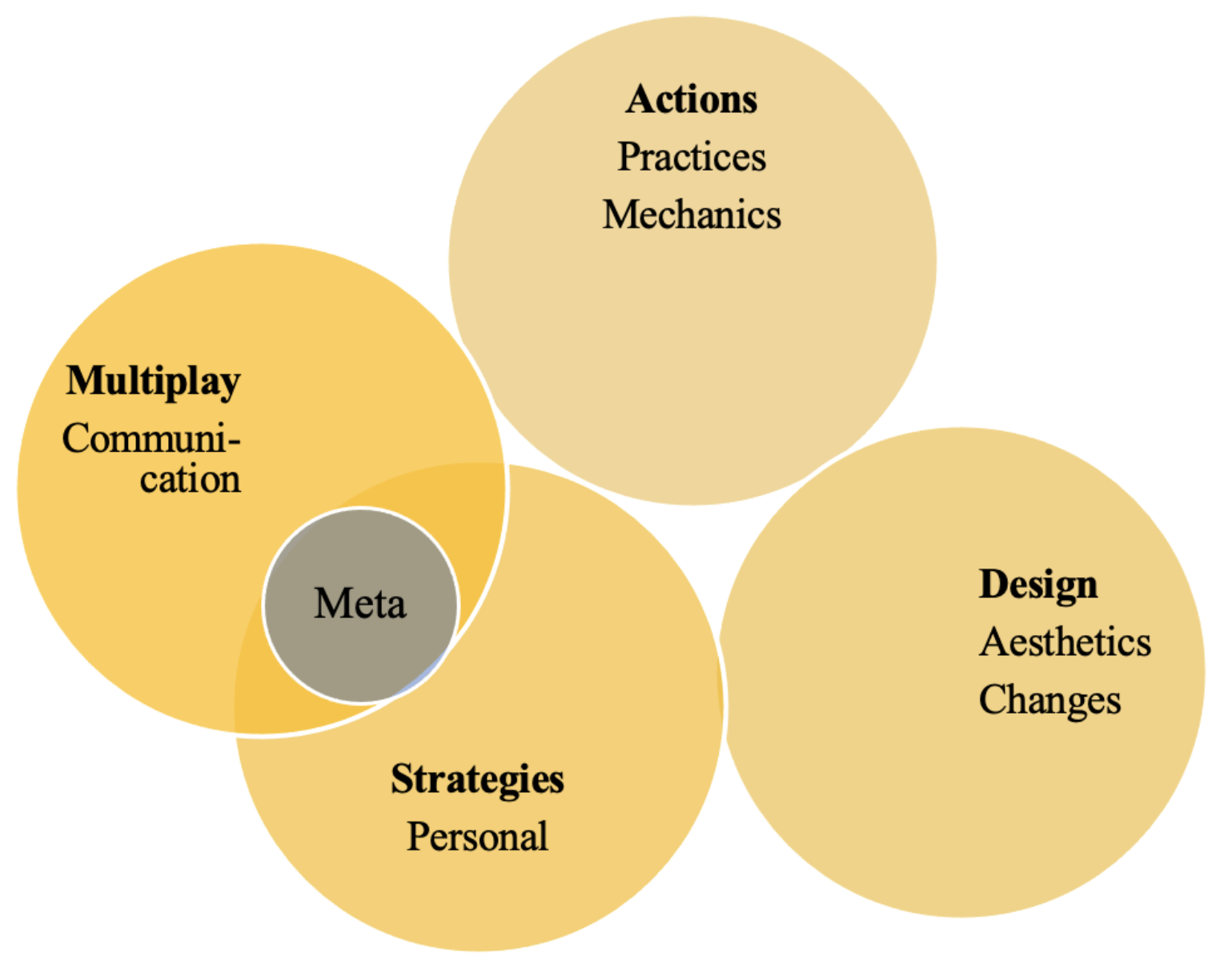

Figure 2. The exploratively formed structure of Among Us, based on the case study.

The above macro-level thematic structures illustrate various abstract differences between the two videogames, as evidenced via the observations of 38 individual ethnographers. We will discuss these macro-level differences below; however, before that, to test the analytic flexibility of the method, we additionally report two micro-level themes that did not surface as dominating categories. Because our analysis did not pursue answers to any research questions (but we carried out open data collection and coding), various potential thematic clusters that might yield specific insight were left ungrouped and unanalysed. By applying an alternative analytic approach, we screened the codes concerning Cyberpunk 2077 with a preselected narrative or storyworld element 'society', and the codes concerning Among Us with a preselected strategic element 'social manipulation' (both elements surfaced as potential micro themes in the above exploratory analysis). Of note, a more detailed analysis and view of the 'society' in Cyberpunk 2077 and 'social manipulation' in Among Us would have been possible if such research questions had motivated this study from the beginning, enabling the ethnographers to focus on these elements and the coders to organize the data based on such 
hypotheses. A complete list of codes that were identified by these 'society' and 'social manipulation' elements is found in Appendix 2.

\subsubsection{Micro example 1: Society in Cyberpunk 2077}

Cyberpunk 2077 is an open world roleplaying game in a science fiction setting, thus providing an opportunity for analysing its represented society in more detail. On a general level, the ethnographers' descriptions of the videogame's society, mainly in Night City, described 'ruthlessness', which was present on various societal levels from (lacking) health care to rampant corruption and violence. According to one ethnographer, 'The citizens have normalized violence and corruption to such a high degree that advertisements must be extreme to attract any attention. The newest porn, penis extensions, and strip clubs are present everywhere.'

Night City has no conventional class structures, but hierarchies are common. Power is distributed among various 'tribes': for instance, corporations, gangs, and the police. These 'tribes' often have conflicting interests, which leads to violent interactions and an overall instability of the society for an individual. Ironically, the videogame presents itself to the player as a meritocracy in the sense that working (playing) more will increase the protagonist's status - capital, networks, skills - almost without exception. Meanwhile, one ethnographer noted: 'The corporations have taken over Night City and deprived the citizens of the two things that capitalism loves most: freedom and choice.'

Many details regarding the daily actions and events in the society were documented. For instance, one observed how the local taxis offer an 'exclusive' service, with which all body parts can be collected automatically in case of an accident. Another recounted an event about a character's mother dying at age 45 due to lacking funds in private healthcare. The following anecdote, in turn, concerns events that took place in space:

The European Space Agency messed up their operations in the solar system. They didn't treat their workers humanely, which led to the O'Neill colonies becoming the first independent ones outside Earth. The workers' peaceful demonstrations were responded to by violence; however, the workers outnumbered them and took power. 
On several occasions, the ethnographers drew links between the society of Cyberpunk 2077 and real-life events, especially in the USA. The political and questionable uses of technology as well as financial power were frequently discussed, for example, as follows:

Society feels like a realistic caricature of the present reality. The rich are richer than ever before, and the poor represent the majority. Money is all that matters: it allows one to buy legal protection, social status, friends, partners, and even eternal life.

Finally, some players also felt that they could change the society or at least have a small impact on it. By following the storyline and completing missions, the player has agency to make Night City a slightly better (or worse) place to live. For instance:

I returned to an area where I had previously completed a small side job of clearing a basketball court of gang members. Now walking through the area, it seems a bit cleaner and is filled with civilians, both just casually moving through the alleyway and playing basketball. I guess I am making a difference in the game world by doing these side jobs.

In the second case study, we move to examine social manipulation in Among Us.

\subsubsection{Micro Example 2: Social manipulation in Among Us}

Among Us is a multiplayer game where one player (the 'imposter') tries to eliminate others ('crewmates') without revealing their identity; thus, it is an opportune title for social analysis. Indeed, various types of deception, lying, and misleading others were documented, representing social manipulation in many ways. Considering the videogame's design, which provides very few other means that can contribute to winning, in one sense social manipulation surfaced as a 'core mechanic', albeit without any clear identity of what it may include or exclude. For instance:

You can also report a body if you are the killer, which allows you to move the attention from yourself and convince others of your innocence. 
This one player tries to confuse others by saying 'Don't go there, nothing to see' and 'This would be a perfect place to hide a body.' But the player is a crewmate!

Sometimes staying silent works well, but it can also be interpreted as suspicious.

As an important distinction, social manipulation took place in both spoken (audio) and written (typed) formats. In the audio channels, players could use their own tone or voice as an instrument; for instance, one reported, 'The player spoke very fast from the start and maintained it all the way to the end, which I believe was a strategy that helped them win.' In text communication, in turn, other strategies emerged, such as, 'This one fellow tries to manipulate us by repeatedly asking what the red text means.' Some types of manipulation were not dependent on the media, however.

It is common to admit the possibility of being guilty, and then set up a defence.

Sometimes players launch a meeting immediately so that they can vote out the player they don't like. It's surprisingly easy to get others to follow you.

The more you work to earn the trust of others along the match, the easier it is to control the end game. They believe anything you say.

The latter aspect of social manipulation - earning trust - was likely the most dominant in general. In many contexts, the ethnographers described trust as playing a key role, and players used diverse strategies for luring others to trust them. The below anecdote is illustrative:

I was the imposter, but I acted as I would be protecting a crewmate, as you would normally do when playing as a crewmate. I could thus make other players believe that I am harmless, and later abuse that trust by keeping it going until the win. 
Some journals had also documented 'metaleptic' (see Kukkonen \& Klimek 2011) strategies, which occurred at the crossroads of technology and the virtual world. The remote act of play created further opportunities for social manipulation:

One player claimed that the battery of the earphones ran out and they had to go change it, due to which they could not be the imposter. This worked, as players didn't vote for them... but it turned out to be a deception in the end.

In the next section, we return to analyse and discuss the macro-level structures of the two videogames in more detail.

\subsection{Discussion: Multiverses of Cyberpunk 2077 and Among Us}

The two studied videogames are radically different by design, and our findings reflect these differences explicitly. None of the large thematic categories (below in bolded capitals) overlapped between these videogames, and while some similarly titled sub-themes (below in bolded) were found in both games (aesthetics, mechanics), their content, as represented by distinct codes (below in italics), still voiced differences, as we explain later. This discussion has three sub-sections, which illustrate the key macro-level differences.

\section{Mechanics and actions}

In both Cyberpunk 2077 and Among Us, a clear thematic cluster emerged around 'what the players do'. However, we found significant differences in how these clusters formed and what they included.

In Cyberpunk 2077, the related observations documented in the ethnographers' journals were often formulated around the idea of mechanics as 'verbs' (Järvinen 2008): that is, collecting, hacking, shooting, skill-tree use, upgrading, and so on. We classified these verb-based codes into a distinct interactions sub-theme, which was situated in two larger categories of Mechanics and Storyworld due to these verbs strongly contributing to both. Two other subthemes belonged to the Mechanics category as well: bugs and play styles. Several codes, such as a car went through a wall and dialogue turned Polish represented the bugs sub-theme, which was located under the larger categories of Mechanics and Aesthetics due to such glitches often 
surfacing in both thematic domains. The third and final sub-theme, play styles, included codes concerning how the videogame was or could be played; for instance, preferred weapons and stealth implied play style options, whereas difficulty referred to how players could also choose their level of challenge. This sub-theme belongs to Mechanics alone.

In Among Us - similarly but not identically to the above - a large thematic category of Actions consisted of various descriptions of what players could and could not do, or what the 'rules' were and how they were (or could be) followed. For instance, codes such as minigames, votes, missions, reporting corpses, and the dead cannot converse represent the range of potential actions and their limits, which we divided into two sub-themes: mechanics and practices. The former, despite being labelled mechanics, did not include explicit codes of verb-based interactions like Cyberpunk 2077 (one exception: killing), but rather they are more general principles of action and doing, such as the above minigames and vote, or spectating and loss. In turn, practices include more event-like codes such as the above missions and the dead cannot converse, or chat discussing imposter and getting caught.

In sum, despite referring to the same areas of content ('what the players do'), the thematic category of Actions in Among Us differs from the Mechanics in Cyberpunk 2077 by lacking clear distinguishable interactions and instead being more descriptive of what is 'going on' or 'happening' when players play. We interpret this as a reflection of Cyberpunk 2077 representing canonized open world roleplaying (see Hitchens \& Drachen 2009), whereas Among $U_{s}$ does not seem to fit any conventional genre (e.g. Steam classifying it as 'casual' and Wikipedia as 'party'). The history of roleplaying videogames is strongly tied to the evolution of diverse game mechanics (see Zagal \& Deterding 2018), which likely is also shown in Cyberpunk 2077's structure and play experience: many of the interactions that have been designed and that players engage in are 'game mechanics' in the generic sense (see Gregersen 2014). Nevertheless, in Among Us - although it naturally involves various 'game mechanics', too - players spend a lot of time interacting in ways that are less mechanical or mechanical in a different way: for instance, talking to other players and thinking about what they are planning, which might be better described as specific design patterns (Björk \& Holopainen 2005). These findings suggest that scholars interested in 'what players do' in videogames may need more complex (inter)action models than those based on 'game mechanics', especially in studies outside conventional genres. 


\section{Aesthetics and design}

In Cyberpunk 2077, a cluster of observations formed a larger theme of Aesthetics. In addition to the previously noted bugs, two other sub-themes were identified: audio-visual and tech motif. The former mainly includes codes regarding the visual and sonic elements but also stylistic details, such as beautiful streets, and detailed graphics, fitting music in different places, sex ads, and violence. The latter consists of codes about the specific motif of technology, which the ethnographers had observed in many ways, as in artificial intelligence, braindance, cyborgs, futuristic cars, and implants. We classified this tech motif under both Aesthetics and

\section{Environment.}

Although the ethnographers made aesthetic observations about Among Us, too, these observations were fundamentally different in form and kind. We labelled this macro-thematic category Design, with two sub-themes. The first sub-theme, aesthetics, includes codes that are closer to those of Cyberpunk 2077 - for instance, simple graphics, browser-like game, and comics aesthetics - but also player voice covers game sound and character art customized. The second sub-theme was termed changes, as many codes were dedicated to observations regarding alterations in the design, such as proximity chat mod, new roles, play remains interesting [with changes], jester [modification], and evolving player-created rules.

Despite the ethnographers observing aesthetic elements in both videogames, in Cyberpunk 2077 a large part of these were strongly related to simulation. Several observations about Night City - as a simulated virtual location where Cyberpunk 2077 takes place - were coded; the ethnographers had clearly been influenced and provoked by these elements. Their centrality was highlighted by the numerous instances where they did not meet the players' quality criteria (bugs). In Among Us, on the other hand, very few, if any, coded observations concerned audiovisual simulation. Whenever the visuals were described, the observations were generally abstract (e.g. no useless details), and most of such codes concerned audio (e.g. steps make different sound effects at different locations).

Overall, we interpret these differences in the aesthetic codes as a reflection of the players' experiences; although both Cyberpunk 2077 and Among Us arguably 'simulate' multiple realworld entities (Giddings 2014; Karhulahti 2015; Möring 2013), only in the former do such simulations surface meaningfully to players. Moreover, this lack of meaningful simulation in 
the latter appears to be compensated by differently aesthetic design elements, such as character customization and frequent content changes (see Sihvonen 2011; Wirman 2011). In videogames like Cyberpunk 2077, high-level simulation appears to be expected and contributes to the experience directly, whereas in videogames like Among Us, simulation serves as an 'ornament' (see Eskelinen 2012) for various types of ludic and social interaction that constitute the core of the play experience.

\section{Storyworld and multiplay}

As a story-driven open world roleplaying videogame, Cyberpunk 2077 has a rich storyworld with characters, narratives, and possibilities to interact with them in a large simulated city. Unsurprisingly, many of the ethnographers' observations concerned these elements in particular. We call this thematic category Storyworld, with sub-themes characters and narrative next to the previously mentioned interactions. A large part of the ethnographers' attention was given to the videogame's characters, from simply naming them (Jackie, T-Bone, etc.) to more detailed descriptions, such as multiculturality, Judy's childhood, and chat with a hallucination friend. For the section narrative, we included related descriptions, events, and storylines; for instance, some observations like trip to Arizona and many storylines explicitly commented on the videogame's narrative components, whereas others pointed at their interpretations or related features, as in crimes, developing friendships, and family drama.

As an online videogame without an explicit story, the ethnographies of Among Us did not document any narrative or storyworld elements (one exception: maps include narrative characteristics). Rather, and unlike Cyberpunk 2077, a large part of the code was dedicated to diverse types of social play elements, which we merged into a Multiplay thematic category with communication and meta sub-themes. The former sub-theme includes codes such as teamwork, trust, and slow typing makes it difficult to defend, with some codes also dealing with technical issues, such as forgetting to mute mic and language. The meta sub-theme mainly concerned the agreements or knowledge shared among the community of players (e.g. rules between players, social contracts, and handicap). We positioned this sub-theme in both the Multiplay and Strategies thematic categories.

The above illustrates the major difference between the single-player experiences of Cyberpunk 2077 and multiplayer experiences of Among Us. In the former, the majority of documented 
observations concerned the Storyworld and the Environment, whereas in the latter a significant number of the observations were dedicated to documenting the multiplayer design and interactions with other players. Although this is hardly anything new to the researchers, it further highlights the need for clinical gaming-related health research to not address 'videogames' as a homogenous entity. As our findings evidence, the play experiences of videogames like Cyberpunk 2077 and Among Us are fundamentally different, with utterly diverse interactions and components of potential meaning. Multiverse ethnography seems to be one possible means for distinguishing such differences.

\section{Debriefing and limitations: Multiverse ethnography as a method}

Multiverse ethnography is a promising methodological direction with significant potential as well as limitations. We discuss four reflections and related recommendations.

Diversity of voices. In this study, we had 38 ethnographers, each of which spent a minimum of 30 hours with their chosen videogame. This adds up to some 1140 hours of fieldwork, which is not a large sum by ethnographic standards; yet, instead of consisting of a single deep perspective, it provides multiple (narrow) perspectives. In the future, this should be taken into consideration when choosing the object of study. Depending on the team's size and resources, scholars should carefully assess their research question; for instance, in a videogame like Cyberpunk 2077, 30 hours is unlikely enough to complete the main storyline, let alone other explorable content. Our results are thus biased in terms of what is provided by the first half of Cyberpunk 2077; if we had wanted to gain a better understanding of the latter half, the 1140 hours could have been better allocated (e.g. to 12 ethnographers with 120 hours each), thus trading breadth for depth. We recommend those interested in applying multiverse ethnography to carefully evaluate their specific needs.

Consistency of voices. The key strength of the method is also its weakness: the more researchers there are documenting observations, the more difficult it is to maintain consistency in reporting. Our case studies were conducted with BA and MA students who had varying experience with qualitative methods, the studied videogames, and culture in general. This likely contributed to both the richness of data (many unique perspectives) as well as unreliability (some codes and journals were difficult to analyse and interpret). For future multiverse ethnographies, we recommend the use of a standardized format for fieldwork journals across participating 
researchers, including a synchronized style of writing - for instance, paragraphs by topics with bold and italics used for highlighting - with pre-planned (but flexible) fieldwork journal length. This will be useful later in the coding process, balancing individual stylistic differences in writing, which can produce bias in overall data representation unless carefully controlled. ${ }^{1}$

Research questions and manuals. Our case studies did not have an explicit research question. Still, even the openly documented and coded data yielded interesting findings, as we screened it exploratively for results regarding 'society' (Cyberpunk 2077) and 'social manipulation' (Among Us). This is encouraging for future work, which should apply multiverse ethnography to specific research questions instead of conducting open studies. Even when scholars are interested in mapping overall ontological dimensions (e.g. Therrien 2017; Zagal et al. 2007) or the challenge structures (e.g. Bowman et al. 2021; Vahlo \& Karhulahti 2020) of a chosen videogame (or technological artefact), we recommend providing a guiding manual for the ethnographers, which can be supplemented by other areas of possible research interest. Although our open approach produced an impressively diverse pool of observations, in more than one instance it was arguably too diverse, as evidenced by many subjective opinions, which we had to exclude as irrelevant. By providing the ethnographers a manual with the areas of interest systematically outlined before they start fieldwork - without compromising explorative power, if that is the goal - the results will be more focused and contribute directly to the research question, also saving valuable resources.

Principal-associate design. In our case studies, each ethnographer worked independently and openly, thus producing fully autonomous observational accounts. For many research questions, however, it is likely more efficient for one researcher to carry out deeper initial fieldwork (e.g. for 150 hours), based on which multiple ethnographers can be recruited to validate tentative observations, fill in discovered 'gaps', or expand specific areas of interest with a more focused approach (e.g. for 50 hours). The evident limit here is that access to some videogame areas or domains of related knowledge may require significant effort and time that cannot be 'skipped'; to answer research questions concerning such data, other methods might be more fitting. If

\footnotetext{
${ }^{1}$ Our fieldwork journals were 2-58 pages long. In order to control bias, we balanced these major differences by trimming the number of codes and themes in the analysis phase (i.e. a large number of codes could be marked for a single paragraph in a shorter journal, whereas in longer journals codes were chosen more selectively). Of course, this did not completely even out the differences.
} 
multiverse ethnography is applied in such a case, scholars should rely on fewer but more committed long-term fieldwork accounts.

\section{Conclusions}

In this article, we have presented multiverse ethnography as a systematic qualitative method for gaming and technology use research. In two preregistered case studies with 38 unique ethnographers, we applied the method to Cyberpunk 2077 and Among Us to test the operability of multiverse ethnography in practice. With open data collection and analysis, our results evidenced and structurally organized design and experiential differences, three of which are summarized below:

a. What is often simplified as 'game mechanics' seems to apply to genre-based videogames like Cyberpunk 2077 relatively well - players clearly recognize such mechanics and consider them important - yet in videogames like Among Us, players instead seem to perform and enact various 'actions' or 'practices' that describe more generally the events in which people participate.

b. Although videogames are often considered 'simulations', our findings reflect a significant difference in how players of different videogames experience simulation. In Cyberpunk 2077, simulated entities and events were the dominant documented objects, whereas in Among Us such elements (despite being present) were ignored almost completely, the focus being rather on the 'functional aesthetics' of design.

c. Both Cyberpunk 2077 and Among Us include several unique features that did not surface in the documented observations of the other. This reaffirms the diversity of experiences that different videogames provide for their players, ultimately highlighting the need for researchers to identify the objects of study in detail when referring to their usage.

Our work confirms the possibility of using multiverse ethnography as a method in future research - however, with caveats and limitations. We recommend future applications of multiverse ethnography to either start with a clear research question as a filter or use existing design ontologies; both of these can enable ethnographers to better coordinate and synchronize their observations. We also encourage teams to carefully plan the distribution and documentation of fieldwork, as different technological artefacts and videogames require 
different types of engagement to be properly understood. In cases when the objects of research are not well known beforehand, the 'principal-associate' design appears promising by allowing one researcher to chart the field and dynamically define the need for future observations.

Multiverse ethnography with a large team can be a useful approach, especially when the object of study is not designed to be used for thousands of hours; for instance, large virtual worlds may benefit more from fewer and deeper ethnographies, whereas the understanding of less sizable titles could be significantly widened by a greater diversity of perspectives. As a final point, we emphasize the possibilities of multiverse ethnography outside gaming. Studies concerning, for instance, specific types of social media or online platforms could benefit from the multivocal qualitative insight that multiverse ethnography provides. Considering the numerous limitations of this initial investigation, we also look forward to criticisms and revisions that hopefully empower and support further methodological development.

\section{References}

Aarseth, Espen and Grabarczyk, Pawel (2018), 'An Ontological Meta-Model for Game Research', in DiGRA Proceedings '18, Turin, Italy, 25-28 July, DiGRA.

Arjoranta, Jonne and Karhulahti, Veli-Matti (2014), 'Ludology, Narratology and Philosophical Hermeneutics', in Proceedings of Nordic DiGRA 2014, Visby, Sweden, 29-30 May, 2014, DiGRA.

Atkinson, Paul and Hammersley, Martyn ([1983] 2007), Ethnography: Principles in Practice, 3rd edn, London: Routledge

Björk, Staffan \& Holopainen, Jussi (2005) Patterns in Game Design. Hingham: Charles River. Boellstorff, Tom (2008), Coming of age in Second Life: An anthropologist explores the virtually human. Princeton: Princeton University Press.

Boellstorff, Tom, Nardi, Bonnie, Pearce, Celia, and Taylor, T.L. (2012), Ethnography and Virtual Worlds: A Handbook of Method, Princeton: Princeton University Press.

Bowman, Nicholas, Lin, Jih-Hsuan Tammy and Wu, Chieh (2021), 'A Chinese-Language Validation of the Video Game Demand Scale (VGDS-C): Measuring the Cognitive, Emotional, Physical, and Social Demands of Video Games', in CHI Conference on Human Factors in Computing Systems (CHI '21), Yokohama, Japan, 8-13, May, 2021, New York: ACM.

Braun, Virginia, and Clarke, Victoria (2006), 'Using thematic analysis in psychology', Qualitative research in psychology, 3:2, pp. 77-101. 
Campbell, John L., Quincy, Charles, Osserman, Jordan and Pedersen, Ove K. (2013), 'Coding in-depth semistructured interviews: Problems of unitization and intercoder reliability and agreement', Sociological Methods \& Research, 42:3, pp. 294-320.

Chen, Mark (2012) Leet Noobs: The Life and Death of an Expert Player Group in World of Warcraft, New York: Peter Lang.

Consalvo, Mia (2020) 'Kaceytron and transgressive play on Twitch.tv', in K. Jørgensen and F. Karlsen (ed.), Transgression in Games and Play. Cambridge, MA:MIT Press, pp. 83-98

Consalvo, Mia \& Dutton, Nathan (2006) 'Game Analysis: Developing a Methodological Toolkit for the Qualitative Study of Games', Game Studies, 6:1.

Dibbell, Julian (1994). 'A Rape in Cyberspace or How an Evil Clown, a Haitian Trickster Spirit, Two Wizards, and a Cast of Dozens Turned a Database into a Society. Annual Survey of American Law, 3, pp. 471-490.

Elverdam, Christian \& Aarseth, Espen (2007) 'Game Classification and Game Design: Construction through Critical Analysis', Games and Culture, 2:1.

Eskelinen, Markku (2012). Cybertext Poetics: The Critical Landscape of New Media Literary Theory. New York, NY: Bloomsbury Publishing USA.

Giddings, Seth (2014) 'Simulation', in M. Wolf \& B. Perron (Eds.), The Routledge companion to video game studies. New York, NY: Routledge. pp. 259-266

Gregersen, Andreas (2014) 'Generic Structures, Generic Experiences: A Cognitive Experientialist Approach to Video Game Analysis', Philosophy \& Technology, 27:2, pp. 159175.

Gruppetta, Maria (2004), 'Autophenomenography? Alternative Uses of Autobiographically Based Research,' in Association for Active Researchers in Education (AARE) Conference Paper Abstracts-2004. Sydney: AARE

Hitchens, Michael, \& Drachen, Anders (2009) 'The many faces of role-playing games', International journal of role-playing, 1:1, pp. 3-21.

Iversen, Sara Mosberg (2012), 'In the double grip of the game: Challenge and Fallout 3', Game Studies, 12:2.

Järvinen, Aki (2007) 'Games without Frontiers: Theories and Methods for Game Studies and Design', Doctoral Dissertation. Tampere: University of Tampere.

Karhulahti, Veli-Matti (2012) 'Double fine adventure and the double hermeneutic videogame', in Proceedings of the 4th International Conference on Fun and Games, Toulouse, France, September, 4-6, pp. 19-26. 
Karhulahti, Veli-Matti (2015) 'Do videogames simulate? Virtuality and imitation in the philosophy of simulation', Simulation \& Gaming, 46:6, pp. 838-856.

Kukkonen, Karin, \& Klimek, Sonja (Eds.) (2011) Metalepsis in Popular Culture, Berlin: de Gruyter.

Linstone, H. \& Murray, T. (2002) The Delphi Method: Techniques and Applications. Originally published in 1975 by Addison-Wesley.

Malliet, Steven (2007) 'Adapting the principles of ludology to the method of video game content analysis', Game Studies, 7:1.

Meier, Adrian and Reinecke, Leonard (2020) 'Computer-mediated Communication, Social Media, and Mental Health: A Conceptual and Empirical Meta-review', Communication Research, 48:8.

Meyer, Michelle N. (2018) 'Practical Tips for Ethical Data Sharing', Advances in Methods and Practices in Psychological Science, 1:1.

Miller, Kiri (2008), 'The accidental carjack: Ethnography, gameworld tourism, and Grand Theft Auto', Game Studies, 8:1.

Möring, Sebastian (2013) 'The Metaphor-Simulation Paradox in the Study of Computer Games', International Journal of Gaming and Computer-Mediated Simulations, 5:4, pp. 4874.

Noble, Helen and Smith, Joanna (2015) 'Issues of validity and reliability in qualitative research', Evidence-Based Nursing, 18:2, pp. 34-35.

Pearce, Celia (2009), Communities of Play: Emergent Cultures in Multiplayer Games and Virtual Worlds, Cambridge, MA: MIT Press.

Quandt, Thorsten, Grueninger, Helmut, \& Wimmer, Jeffrey (2009), 'The gray haired gaming generation: Findings from an explorative interview study on older computer gamers', Games and Culture, 4:1, pp. 27-46.

Rosas, S. R. (2017), 'Group concept mapping methodology: towards an epistemology of group conceptualization, complexity, and emergence', Quality \& quantity, 51:3, pp. 1403-1416.

Saldaña, Johnny (2012), The Coding Manual for Qualitative Researchers, 2nd ed., London: Sage.

Sihvonen, Tanja (2011) Players Unleashed! Modding The Sims and the Culture of Gaming. Amsterdam: Amsterdam University Press.

Snodgrass, Jeffrey G., Bagwell, Andrew, Patry, Justin M., Dengah, HJ Francois II, SmarrFoster, Cheryl, Van Oostenburg, Max, and Lacy, Michael G. (2018) 'The partial truths of compensatory and poor-get-poorer internet use theories: More highly involved videogame 
players experience greater psychosocial benefits', Computers in Human Behavior, 78, pp. 1025.

Strauss, A., and Corbin, J. (1994), 'Grounded Theory Methodology: An Overview', in N. K. Denzin, and Y. S. Lincoln (Eds.), Handbook of Qualitative Research, pp. 273-285. Thousand Oaks, CA: SAGE.

Sudnow, David (1983), Pilgrim in the Microworld, New York, NY: Warner Books.

Sundén, Jenny, \& Sveningsson, Malin (2012), Gender and sexuality in online game cultures: Passionate play. New York, NY: Routledge.

Syed, Moin, \& Nelson, Sarah C. (2015), 'Guidelines for establishing reliability when coding narrative data', Emerging Adulthood, 3:6, pp. 375-387.

Taylor, T. L. (2006), Play between Worlds: Exploring Online Game Culture, Cambridge, MA: MIT Press.

Therrien, C. (2017), 'From Video Games to Virtual Reality (and Back). Introducing HACS (Historical- Analytical Comparative System) for the Documentation of Experiential Configurations in Gaming History', in Proceedings of DiGRA '17. Melbourne, Australia, July, 2-6.

Vahlo, Jukka and Karhulahti, Veli-Matti (2020), 'Challenge Types in Gaming: Validation of Video Game Challenge Inventory', International Journal of Human-Computer Studies, 143, 102473.

Wirman, Hanna (2011), Playing the Sims 2: Constructing and Negotiating Woman Computer Game Player Identities through the Practice of Skinning, Ph.D. Thesis, Bristol: University of the West of England.

Zagal, Jose, and Deterding, Sebastian (Eds.). (2018), Role-playing game studies: Transmedia foundations. New York, NY: Routledge.

Zagal, Jose, Mateas, Michael, Fernández-Vara, Clara, Hochhalter, Brian, and Lichti, Nolan (2007), 'Towards an Ontological Language for Game Analysis', in Proceedings of DiGRA '07. Tokyo, Japan, September, 24-28, Available at http://www.digra.org/wpcontent/uploads/digital-library/06276.09313.pdf. Accessed 25 May 2021 


\section{Appendix 1}

\section{A description of Cyberpunk 2077 sub-themes with three examples of each. Translated from Finnish to English.}

Audio-visual: The audio-visual sub-theme includes aesthetic elements created mainly by the graphics and sounds of the game.

Example A: 'In the nighttime, the Night City looks quite impressive. The play of light and shadow is amazing. I wouldn't want to live there myself, though.'

Example B: 'We are in Mikoshi's data fortress, V walks along a kind of pixel bridge with an upward beam of light ahead and Alt quotes some rhyme about the journey, through the streets, as the evening shines in the sky, like a patient on the table, passing through cheap hotels, streets that reminds arguments, leading to the overwhelming question - what should not be asked what it is, but let's go make that visit.'

Example C: 'At first glance, the world feels alive. But when you pay more attention, it's easy to notice that the pedestrians don't do anything special. They just keep repeating the same actions.'

Bugs: Bugs refer to issues within the videogame's code or design, unintended and unexpected by the developers.

Example A 'Some accidental comedy was added when a dead corpse lying on the ground kept confidently yelling how I can't escape from him.'

Example B 'A hideous child splits into three at his upper torso and waves his three pairs of arms around...'

Example C: 'Jackie walked through the car, which probably is a mistake (bug) in the game.'

Characters: Observations on the numerous characters of Cyberpunk 2077. In addition to the key characters, with deep histories and personalities, the sub-theme also includes light NPCs, enemies, and groups of characters like the police. 
Example A: 'The characters seem to really like the city despite the fact they had just carried out an intense job involving death and destruction ... Jackie on the other hand seems quite sensitive and seems to have a sweet side.'

Example B: 'Kerry thinks the girl band 'Us Cracks' has robbed him something and they need to be stopped. Kerry and V go backstage, Kerry threatens the girls with a gun, hates everyone and puffs that rock is dead. V asks what the deal was. Johnny comments amused that the girls have potential. The girls admire Kerry and promise to cancel the tour. The quality of the deal/robbery stays unclear, but Kerry tells the girls they are ok. They take a selfie together.'

Example C: 'I met a preacher on the street. I was able to have a dialogue with the preacher and then two guys arrived and started taking photos together.'

Interactions: How the player interacts with the videogame, either by explicit mechanics or more implicitly exploring the virtual environment.

Example A: 'I think the inventory system is a little bit counter-intuitive and using it is somewhat difficult. Maybe it's just me, but I often struggle with it.'

Example B: 'There is a choice to go to the waterhole and back to the body. The player must make a choice.'

Example C: ' The steal mechanic reminds of Metal Gear Solid V and The Last of Us.'

Locations: Places in the virtual environment, but also observations related to them, such as culture, religion, and laws.

Example A: 'God and Christianity still seem to be present in this world. Sometimes characters say a prayer and some wear cross necklaces. I also read an article about Buddhism while I was visiting Arasaka Tower.'

Example B: 'Advertisements are strongly visible on the streets of Night City. There are billboards nearly everywhere with screens running advertisements. The main tactic seems to be provocation - for me the most unforgettable one was a luxury wear ad depicting a stylish man collapsed down at some alley, with a syringe on his arm. The ad said: At least you're wearing Jinguji.'

Example C: 'There seems to be striptease bars in every street corner.' 
Narrative: The story or storiable elements, often related to quests, missions, and dialogue that the player needs to complete to progress.

Example A: 'The story began a little bit differently than I expected it would. Moving from the wasteland to the city and adapting to it was easier and faster than I expected. On top of that, you weren't really allowed to play it. Instead, the whole scenario was just presented as a compilation of cutscenes.'

Example B: 'Dex shoots $\mathrm{V}$ at the end and after this comes an impression of death and Game Over. But then we are in the hall of some building where we meet a girl and a man. The hall leads to the backstage. In the mirror image we see that the avatar has changed from $\mathrm{V}$ to Johnny Silverhand, played by Keanu Reeves.'

Example C: 'Information gathered in a previous side quest led to another side quest. Same characters/gangs stay in the story, which adds personal touch to the quests. Things like these bind the story nicely together.'

Play styles: This sub-theme focuses on the players' strategies, skills, and choices relevant to play. Also includes notes about what was found challenging or worth observing in their own choices.

Example A: 'I use a silenced pistol, and thanks to the skills I've chosen, it very efficiently kills enemies in one shot. When the enemies spot me, I switch to melee using a samurai sword.' Example B: 'On the map of the city there are several jobs or gigs to be chosen from, and even more cars to buy. I cannot learn what the payment for a job is because the players I observe are very quick in their movements. RR already has several vehicles in his inventory, so I can't figure out whether he has bought them with money earned or if he has been using some kind of cheat for endless money.'

Example C: 'It's difficult to say how interesting character improvement is when you're not actually experiencing the game mechanics yourself.'

Tech motif: Aspects in the storyworld regarding technology. This includes several mentions about futuristic and science fiction themes as part of the virtual environment.

Example A: 'They talked about cyber implants in the news. With them people can transfer their soul from a dying body to a new one and thus reach immortality.' 
Example B: ' 'Jackie hasn't altered himself much, but for example Dum Dum has been enhanced so much he is almost unrecognizable as human.'

Example C: 'The tutorial was shown in a so-called VR environment, but instead of putting the glasses on, $\mathrm{V}$ inserted a microchip inside the back of his head. There is a wire coming out of V's wrist that can be connected to the computers and with which one can read other people's systems (cybernetics).'

\section{A description of Among Us sub-themes with three examples of each. Translated from} Finnish to English.

Practices: How the videogame works and is played; the actions that different player roles are supposed to take in order to win.

Example A: 'The imposter's loop between sabotage and murder and the crew's repair tasks still make up the core of the game.'

Example B: 'The game doesn't seem to guide much but only lets you know the goals, so crewmates do the missions and the impostor tries to kill the others. So the game doesn't seem to offer a way to play it, even if the winning conditions are set on the table. Everyone can make the game as they want, and the experience can vary in different ways.'

Example C: 'The voice chat works with friends and strangers.'

Mechanics: Various in-game and out-game mechanics with observations of their roles, such as how players use cameras, Discord, or hacks.

Example A: 'There were too many camera campers in our group. It made killing too difficult and winning by completing tasks became impossible.'

Example B: 'After the game was started, the streamer went through the functions of the game mechanics practically in body reporting as well as in common practice of how they talk to each other during the game, when usually players can only use text to communicate during meetings.'

Example C: 'I sought players from Discord and we started a group call, same rules as last time. This time I wanted to try camera observation, however.'

Meta: Commonly applied tactics and terminology used to communicate them. 
Example A: 'The phrases I saw him enter a vent and if it's not them then vote me still work every vote.'

Example B: 'One of the most effective lies in my experience is to frame someone else for a kill you did.'

Example C: 'Often used strategies: double kill, accusing others, alibi, using vents (after a kill in particular), walking in pairs, and checking spaces systematically.'

Communication: Slang and lingo, the role of communication in the play experience. How playing with friends or strangers affects communication, and so on.

Example A: 'If the lobby was mostly silent, talkative players were suspected. If the lobby was filled with guys with silly usernames memeing around, the more serious players were thrown out.'

Example B: 'In terms of identity, the visual side is certainly not the only way to express identity. Personal communication plays such a big role in the game.'

Example C: ' In one match there was only three of us left... and well just stood still, staring. Others who had been eliminated just laughed and joked about Mexican Standoff.'

Personal strategies: Tactics or strategies on a personal level, not necessarily common or known in the community.

Example A: 'I examined how well the if it's not them vote me strategy works. At first, I used it only if I was completely certain about an impostor's identity. Almost every time the other crew members were convinced, and the impostor was dealt with.'

Example B: 'In the videos I watched, I noticed how some strategies may be risky to implement, but when they work, they can be effective. Among other things, the strategy in which the Impostor-player claims that an innocent player jumped on the air duct can at the same time increase the credibility of his own innocence, if others believe the explanation, but this can also easily reverse the conversation direction to accusations of the opposite situation.'

Example C: 'As a crewmate he just spent all time in the camera room and watched others. I asked why he did this, and he told me that whenever playing with his little brother, he does this because the brother cannot do the missions right; thus, the only working tactic for winning would be to catch the impostor.' 
Aesthetics: The graphical or visual outlook of the videogame, but also aesthetic notes about audio and other design components.

Example A: "The first match I watched was streamed during Halloween, so there were appropriate clothing items in the game. The map was also decorated with spider webs, pumpkins, and so on.'

Example B: 'The game is visually very simple, all players can be recognized easily by name, character color and accessories. Players can customize characters simply. Also the environment and the mini-games follow a unified comic-like style, where there are no unnecessary details.' Example C: 'All problems derive from the poor design of communication features and the limited time to use them. A game that is based on social play suffers from lacking audio, such as voice use in normal matches.'

Changes: Notes about how the videogame has changed through updates and patches as well as mods created by the community.

Example A: 'Ultimately, Among $U s$ is a very simple game, and all these mods add some nice challenge to it. Playing the base game is entertaining, but the modified rules make it more fun.' Example B: 'I noticed they were playing a modified version, which had added new roles to play, as in Mafia or Werewolf.'

Example C: 'In the Jester Mod the goal is to act suspiciously and get voted out. It feels quite hard, as you need to balance between getting suspected and not being too obvious.' 


\section{Appendix 2}

\begin{tabular}{|c|c|}
\hline Society (in Cyberpunk 2077) & Social Manipulation (in Among Us) \\
\hline Dystopia & Lying to strangers is easier \\
\hline Only the rich can afford healthcare & Changes in behaviour are easily noticed \\
\hline The line between human and machine & Accusations are often believed \\
\hline The strong rule the world & Slow writing makes defence difficult \\
\hline Uneven distribution of wealth & Players are accused for next to no reason \\
\hline Social criticism & Silence is suspicious \\
\hline Social structure & Following others is suspicious \\
\hline Expensive healthcare & Passivity keeps alive \\
\hline Uncertain security situation & Suspicion (general) \\
\hline Visibility of sex & Exploitation \\
\hline Homelessness & Feint \\
\hline Eating same food unites the rich and the poor & Deceive \\
\hline Traditions & Persuasion \\
\hline Corporate power & Convince \\
\hline The power of money & 'Sus' about suspicious players \\
\hline Guns mean power & Impostors deceive others with explanations \\
\hline Distribution of power & Lying impostors \\
\hline Propaganda & Impostors may be recognized from their talk \\
\hline Multicultural encounters & Appearing as a noob \\
\hline Anarchists & Secret communication between impostors \\
\hline Authoritarianism & Change in style can reveal impostor \\
\hline Propaganda & By talking too much, don't others talk \\
\hline Technological advancement & Talking style affects credibility \\
\hline Racism & Cheming \\
\hline
\end{tabular}




\begin{tabular}{|c|c|}
\hline Waste issues & Killing others in secret \\
\hline Ecological sustainability & Manipulation \\
\hline Cultural stagnation & Coverstory \\
\hline Culture and environment & Natural lie is a significant advantage \\
\hline Worldview & Deception \\
\hline Multiculturalism & Alibi \\
\hline Capitalism & Expressing oneself \\
\hline From utopia to dystopia & Convincing others \\
\hline The decrease of population & Good liar \\
\hline Social and infrastructural issues & Speaks with even voice \\
\hline Violence and corruption are normal & Quiet, doesn't pull attention \\
\hline \multicolumn{2}{|l|}{ Money drives everything } \\
\hline \multicolumn{2}{|l|}{ The gap between the rich and the poor } \\
\hline \multicolumn{2}{|c|}{ Dirt and the poor are hidden from the rich } \\
\hline \multicolumn{2}{|l|}{ The homeless live on trash } \\
\hline \multicolumn{2}{|l|}{ The police are useless } \\
\hline \multicolumn{2}{|l|}{ Dirtiness and unsustainability } \\
\hline \multicolumn{2}{|l|}{ No recycling } \\
\hline \multicolumn{2}{|l|}{ Throw-away society } \\
\hline \multicolumn{2}{|l|}{ Class warfare } \\
\hline \multicolumn{2}{|l|}{ Advertisements represent the society } \\
\hline \multicolumn{2}{|l|}{ Urban decay } \\
\hline \multicolumn{2}{|l|}{ Inequality } \\
\hline \multicolumn{2}{|l|}{ Dependency on technology } \\
\hline \multicolumn{2}{|l|}{ Corporate power } \\
\hline \multicolumn{2}{|l|}{ Recession } \\
\hline Social class & \\
\hline
\end{tabular}


Disregard of human life

First world problems

Traditionalism 